\title{
Estimation of Welding Voltage Using Neural Network in GMA Welding*
}

\author{
by YAMANE Satoshi**, KOIZUMI Manabu***, IMAI Yuusuke***, KANEKO Yasuyoshi** and OSHIMA Kenji**
}

\begin{abstract}
Control of the distance between the tip of an electrode wire and a base metal is important to obtain a good welding quality in spite of an arc fluctuation. Therefore, it is necessary to estimate the welding voltage relating to its distance in not only the steady state but also the transient state. For this purpose, this paper proposes neural network models which output the present welding voltage from the data relating to wire melting, such as past current, past voltage and past wire feed rate. Since performance of the neural network model depends on threshold functions, authors investigated the performance of the neural network models based on both sigmoid function and radial base function. To confirm the validity of these systems, fundamental experiments were carried out. In this paper, performances of the neural network were investigated in pulsed current welding and switch back welding the output data from the neural network were compared with the measured data. It was found that the neural network model based on the radial base function is useful than the sigmoid function to estimate the welding voltage in the switch back welding because of better responses in the transient state and smaller steady state error.
\end{abstract}

Key Words: Neural network model, Estimation of torch voltage, GMA Welding, Wire Melting Phenomena, Arc Characteristics

\section{Introduction}

Recently, due to the decreasing and aging of skill welders, the development of robot-controlled automatic arc welding has been encouraged. With automatic welding, accurate sensing of the welding phenomena is particularly important ${ }^{1.22}$. Consequently further improvements in the accuracy of sensing and advanced performance are highly desirable to achieve better quality welding. It is important to control arc length to get the high quality welding. Since there is some disturbance, such as unstable arc, during the welding, the measurement of the welding voltage with proportion to distance between a tip of the electrode wire and base metal may be difficult. To solve this problem, an intelligent filter was proposed ${ }^{3)}$. In this filter method, before the welding, so many times experiments are needed to adjust the filter. We proposed the estimation method based on neural networks to the electrode wire ${ }^{4}$. In this method, the training data were composed of several kinds of the fundamental experiments. If the estimation error became big, new training data were required. But, the extension can be estimated by using the welding voltage, the current and the wire feed rate. In this paper, we propose the estimation method of the welding voltage based on neural network, which uses the sampled data of welding current, voltage at the current pick-up point, and wire feed rate. That is, the present welding voltage is estimated by using the prior welding voltage, the current and the wire feed rate. In conventional welding for the same work pieces, the welding conditions and the

\footnotetext{
*Received: 2008. 11. 18

**Member, Graduate School and Science of Engineering, Saitama University

***Student Member, Graduate School and Science of Engineering, Saitama University
}

gap are kept constant. The training data for neural network to estimate the welding voltage are made during the begging of the welding in steady state. The neural network may be trained on real time. After that, the welding voltage is estimated by using the trained neural network under the same welding conditions.

In this paper, two kinds of transfer functions, sigmoid function and radial base function, were applied to estimate the welding voltage. The performance of the estimation was examined by using the conventional welding method and the switch back welding method ${ }^{5}$. Because, in the switch back welding, the arc phenomena in slow speed movement are considerably different from that of the high speed movement. The estimation of the welding voltage becomes difficult. The possibility of the neural network as the sensor was considered. The performance of the neural network was verified for the estimation of the welding voltage.

\section{Neural network model}

\subsection{Modeling of welding circuit}

In GMA welding, relationship between the melting phenomenon of an electrode wire and behaviors of arc length has been analyzed ${ }^{6}$. According to this result, inputs of the neural network are determined. Welding circuit was shown in Fig.1. When the wire extension $y$ was approximated in one dimension coordinate, behavior of the extension length $y$ was given by

$$
\frac{d y}{d t}=-v_{m}+v_{f}
$$

where $v_{m}$ is the melting rate of the wire and $v_{f}$ is the wire feed rate. According to wire feed rate of Halm $\varnothing \mathrm{y}^{7}$, Eq.(1) is rewritten by

$$
\frac{d y}{d t}=-\alpha i-\beta i^{2} y+v_{f}
$$




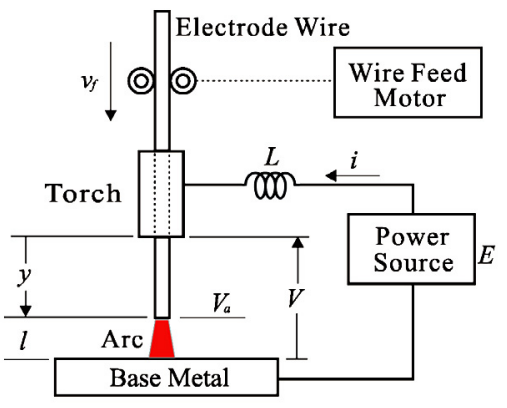

Fig. 1 Welding circuit.

where $i$ is the current and $\alpha$ and $\beta$ corresponds to coefficients depended on the current. First term and second term in right hand of Eq.(2) represented arc heat and Jule heat in the electrode wire, respectively.

On the other hand, let the resistance per unit length in the extension be $R_{0}$. The welding voltage $V$ was given by

$$
V=R_{0} y i+V_{a}
$$

where $V_{a}$ corresponds to the arc voltage. Eq.(3) indicated that the welding voltage depends on the current and the wire extension.

The characteristic of the arc voltage is approximated as the follows

$$
V_{a}=a l+b+(c l+d) i
$$

where $a, c$ and $d$ are coefficients and $b$ is constant.

The variation of the welding current was given by

$$
\frac{d y}{d t}=\frac{E-V}{L}
$$

where $E$ and $L$ are the output of the power source and the reactance of welding circuit including a welding cable, respectively.

\subsection{Inputs and output of neural network model}

According to Eqs.(1) to (5), the welding voltage is found from the current, the wire feed rate and the arc voltage. But, direct measurement of the arc voltage is difficult. If the welding voltage is calculated by using the numerical simulation based on Eq.(1) to Eq.(4), an initial value of the extension length is needed. Since its length cannot be measured directly, the simulation based on Eqs.(1) to (4) becomes difficult.

In order to estimate the welding voltage at the present time, authors used the neural network, of which the inputs are behaviors of the prior welding voltage, the current and the wire feed rate, if the torch height was kept constant.

Sum total of inputs $X$ in each neuron is given by

$$
X=\omega_{1} x_{1}+\omega_{2} x_{2}+\cdots+\omega_{n-1} x_{n-1}+\omega_{n} x_{n}=\sum_{i=1}^{n} \omega_{i} x_{i}
$$

where $x_{i}$ is input and $w_{i}$ is weight corresponding to a synapse, as shown in Fig. 2 (a).

The output of the neuron $\mathrm{z}$ is given by

$$
z=f(X-\theta)
$$

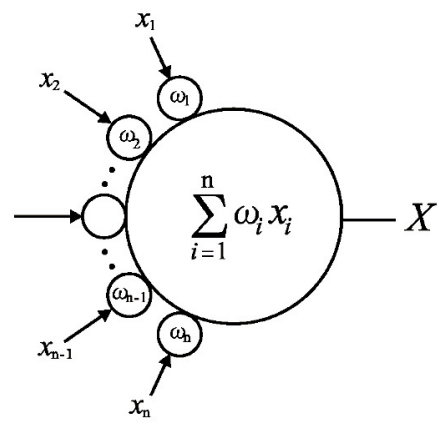

(a)Neuron

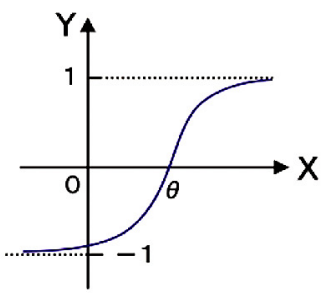

(b) Sigmoid function.

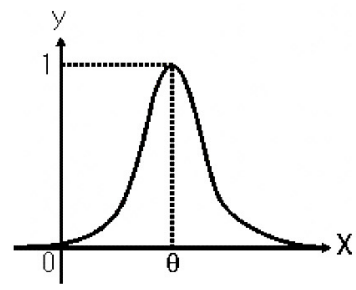

(c)Radial base function.

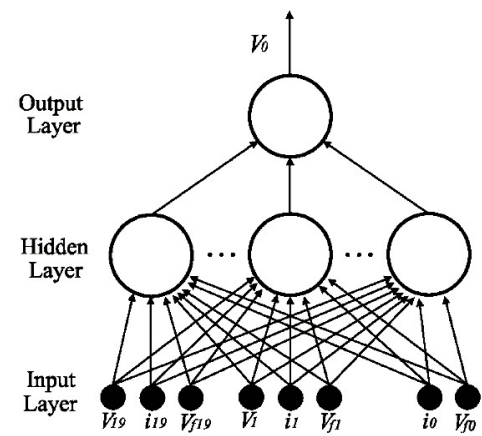

(d)Three layer feed-forward neural network.

Fig. 2 Feed forward neural network.

where $f$ is transfer function and $\theta$ is threshold. $w_{i}$ and $\theta$ are determined in the training of the neural network.

In this paper, two kinds of the transfer function, the sigmoid function and the radial base function, are applied to examine the performance, as shown in Figs. 2 (b) and (c).

The sigmoid function is given by

$$
f(X-\theta)=\frac{1}{1+e^{-(X-\theta)}}
$$

The radial base function is given by

$$
f(X-\theta)=e^{-(X-\theta)^{2}}
$$

It is an advantage of the neural network that input-output relationship can be described for the dynamic state and the steady 
state by training the data ${ }^{8)}$. In this paper, the current, the welding voltage, and the wire feed rate were sampled every $1 \mathrm{~ms}$. The welding voltage, the current and the wire feed rate are selected as one set of inputs to the neural network. For conventional pulsed welding, the frequency of the welding is up to $100 \mathrm{~Hz}$. One period is $10 \mathrm{~ms}$. It was assumed that the transient state of the voltage could be estimated by using the data for $20 \mathrm{~ms}$. Therefore, the data of 20 sets were used for the estimation. But, for the present time, the current and the wire feed rate were used as the set without the welding voltage. The three layer neural network was applied to estimate the welding voltage, as shown in Fig. 2 (d).

The number of the unit in hidden layer was determined from the estimation results in the fundamental experimental results of the pulsed current welding, so that the estimation results becomes good and the estimation results in the sigmoid function is the similar as the radial base function. The input layer and the output layer were composed of 59 units and 1unit, respectively. The hidden layer was composed of 4 units for the sigmoid function and 3 units for the radial base function, respectively.

\subsection{Training of the neural network}

In the constant voltage welding, the fluctuation of the welding voltage is small. But, in the steady state of the arc phenomena, the welding voltage fluctuates in the pulsed current welding than the conventional constant voltage. To examine the performance of the neural network, the authors tried to estimate the welding voltage in the pulsed current welding. The performance of the estimation depends on the training data. The status in the beginning of the welding were given as the training data.

In this paper, two kinds of the training data were used.

1) The conventional pulsed current welding with $60 \mathrm{~Hz}$.

2) The welding voltage in the switch backing welding.

A mixture gas of $\mathrm{Ar} 98 \%$ and $\mathrm{O}_{2} 2 \%$ was used as the

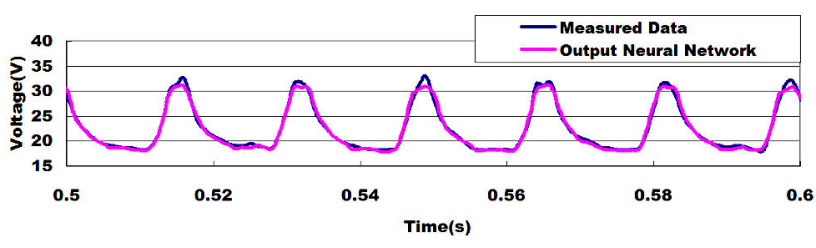

(a)Estimation result of neural network with sigmoid function.

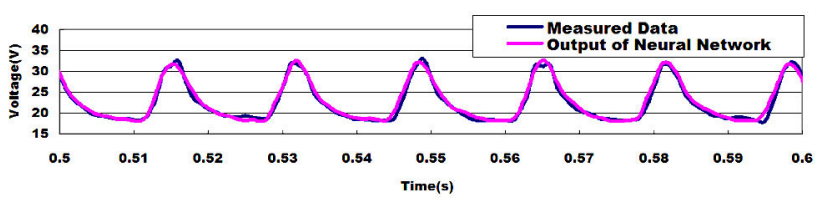

(b)Estimation result of neural network with radial base function.

Fig. 3 Training data in pulsed current welding. shielding gas. In the pulsed current welding, the welding voltage fluctuates due to the arc phenomena and the status of the weld pool. In the switch back welding, the arc phenomena and the status of the weld pool were changed due to the high speed torch movement and the slow speed torch movement.

\section{Estimation result of the welding voltage and discussion}

\subsection{Training and testing in pulsed current welding}

The current, the welding voltage and the wire feed rate in the welding for $0.5 \mathrm{~s}$ to $0.6 \mathrm{~s}$ were selected as the training data, since the transient response is continued up to $0.5 \mathrm{~s}$ from the starting of the welding.

The training results in the neural networks with sigmoid function and radial base function were shown in Fig. 3 (a) and (b), respectively. Since the estimation result is good agreement with the experimental result, a neural network was trained. In the training , the average errors of the estimation in Figs. 3 (a) and (b) are $0.68 \mathrm{~V}$ and $0.99 \mathrm{~V}$, respectively.

The un-training data were used in the checking of the estimation performance. The data at $5 \mathrm{~s}$ after the starting of the welding were used as the testing data. The estimation results are shown in Fig. 4 (a) and (b). The average errors of the estimation in the testing data in Figs. 4 (a) and (b) are $0.45 \mathrm{~V}$ and $0.73 \mathrm{~V}$ respectively. Since the estimation result was a good agreement with the experimental result, a neural network was useful as a model which grasps the action of the welding voltage.

\subsection{Training and testing in switch back welding}

The authors tried to apply the neural network inthe switch back welding for one side back bead welding without backing, as shown in Fig.5. In this welding, the weaving center of the torch repeats a forward and backward like the switch-back movement along the welding direction. That is, two kinds of the travelling speed with $2.5 \mathrm{~Hz}$ weaving, the high speed torch movement and

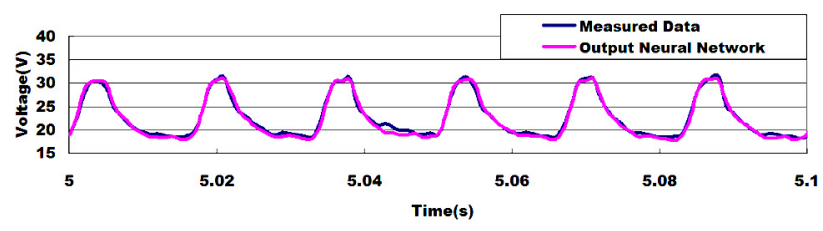

(a)Estimation result of neural network with sigmoid function.

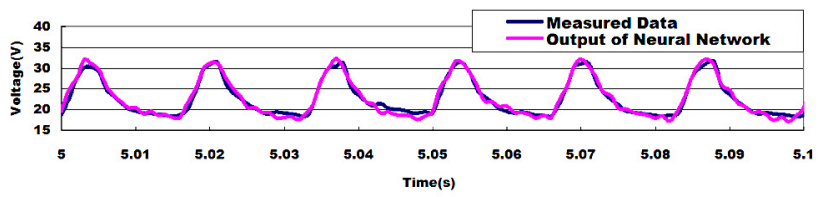

(b)Estimation result of neural network with radial base function.

Fig. 4 Testing result of estimation in pulsed current welding. 


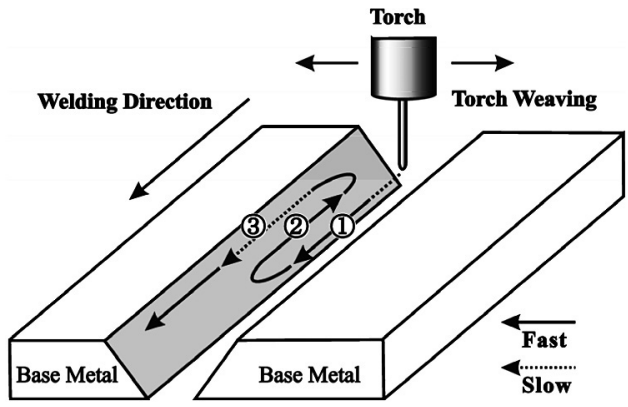

Fig. 5 Switch back welding method.

the slow speed torch movement, were used. The pulsed current was used in the switch back welding. During the torch movement at a high speed, the arc is discharged to the root edge. During the forward movement at the slow speed, the arc heat is given to the weld pool and the surface of the groove, and the bead height is adjusted. According to the temperature rise of the weld pool, its size becomes big. Before the burn through takes place, the torch movement is changed to that at the high speed to take out the heat source from the weld pool.

The current, the welding voltage and the wire feed rate in the welding for $2.6 \mathrm{~s}$ to $8 \mathrm{~s}$ were selected as the training data, since the transient response is continued up to $2.6 \mathrm{~s}$ from the starting of the welding, as shown in Fig.6.

The training results in the neural networks with the sigmoid function and the radial base function were shown in Fig. 6 (a) and (b), respectively. Since the estimation result is good agreement with the experimental result, a neural network was trained.

The un-training data at 13.6s after the starting of the welding were used to examine the estimation performance of the welding voltage. The estimation results are shown in Fig. 7. The estimation of the neural network with radial base function in Fig. 7 (b) was better than the sigmoid function in Fg. 7 (a). This is why the radial base function has an advantage of no overlearning ${ }^{9)}$.

In the sigmoid function, the training of the network was performed by nonlinear transformation and overlearning may take place. On the other hand, in the radial base function, the learning was performed in the small space near the training data and there is no risk for the overlearning. But, the training time of the radial base function becomes longer than the sigmoid function. If the arc phenomena are repeated like the welding, where the constant welding conditions were used, the estimation based on the sigmoid function can be applied.

\section{Conclusions}

The welding voltage was estimated by using the neural network. Two kinds of the transfer function were used in the

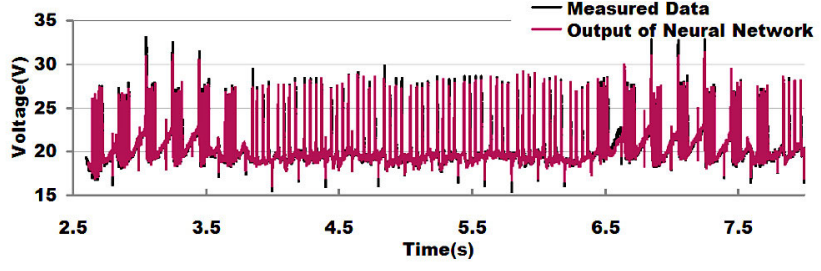

(a)Estimation result of neural network with sigmoid function.

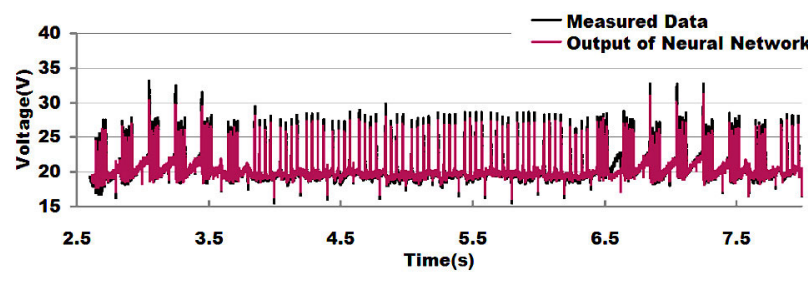

(b)Estimation result of neural network with radial base function.

Fig. 6 Training data in switch back welding.

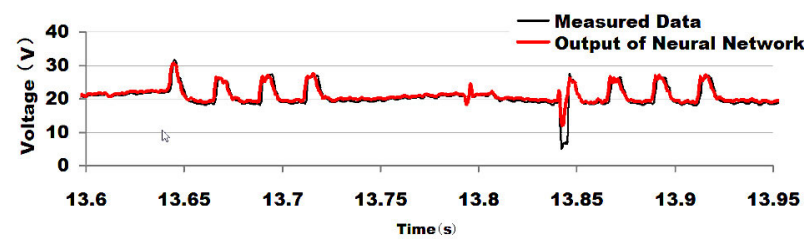

(a)Estimation result of neural network with sigmoid function.

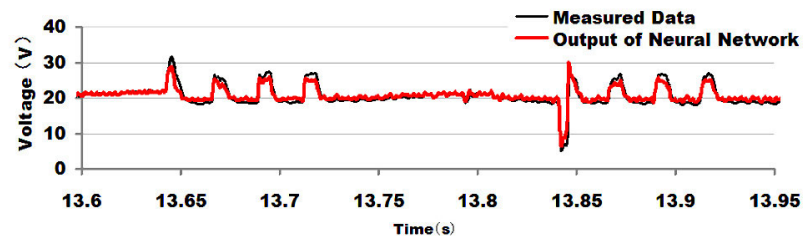

(b)Estimation result of neural network with radial base function.

Fig. 7 Testing result of estimation in switch back welding.

neural network. The estimation results shows as follows

1) When the welding conditions were kept constant during the welding, the estimation performance of the sigmoid function was almost similar as the radial base function.

2) When the welding voltage waveform is greatly changed by the torch movement, the radial base function is better than the sigmoid function.

3) If the training data was composed of the beginning of the welding, the welding voltage can be estimated during the welding.

Furthermore, this neural network may be applied to estimate the actual welding voltage in the welding, in which the arc will be discharged regardless of the shortest distance between the base metal and the tip.

\section{References}

1) Y. Sugitani, W. Mao, M. Ushio, "Adaptive Control of Weld Bead Shape Utilizing Arc Sensor in One Side GMAW Process with 
Backing Plate", Doc. XII-1360-94, pp. 199-206 (1994).

2) P. Drews, G. Starke, "Welding in the Century of Information Technology", Int. Conf. on Advanced Techniques and Low Cost Automation, pp. 3-22 (1994).

3) S. Harada, T. Ueyama: Recent Development of Degital Controlled Power Source, J. JWS, 74-7 (2005), 478-484. (in Japanese)

4) H. Yamamoto, Y. Takano, K. Eguchi, S. Yamane And K. Oshima: Estimation of Arc Length and Wire Extension Using Neural Network, Quar. J. JWS, 20-3 (2002), 378-385. (in Japanese)

5) S. Yamane, H. Yuzawa, Y. Kaneko, H. Yamamoto, M. Hirakawa, K. Oshima: Image Processing and Control of Weld pool in Switch Back Welding without Backing Plate, Quar. J. JWS, 23-1, 65-70
(2005). (in Japanese)

6) Technical Commission on Physics of Welding, JWS: Pulsed Arc Welding, 1986. (in Japanese)

7) E. Halmoy: Wire melting rate, droplet temperature and effective anode heating potential, Conference on Arc Physics and Weld Pool Behavior (1979), 49-59.

8) D. E. Rumelhart, G. E. Hinton, R. J. Williams: Learning internal representations by error propagation, Parallel Data Processing, The M.I.T. Press, 1 (1986), 318-362.

9) K. Levenberg: A Method for the Solution of Certain Problems in Least Squares, Quart. Appl. Math., 2 (1994), 164-168. 